\title{
Research Paper \\ The Effect of Upper Limb Exercise on the Readmission Rate of Myocardial Infarction Patients
}

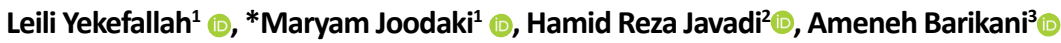

1. Department of Nursing, School of Nursing \& Midwifery, Qazvin University of Medical Sciences, Qazvin, Iran. 2. Department of Cardiology, School of Medicine, Qazvin University of Medical Sciences, Qazvin, Iran.

3. Department of Community Medicine, School of Medicine, Qazvin University of Medical Sciences, Qazvin, Iran.

\begin{tabular}{c|l}
\hline $\begin{array}{l}\text { Use vour device toscan } \\
\text { and read the article online }\end{array}$ & $\begin{array}{l}\text { Citation Yekefallah L, Joodaki M, Javadi H, Barikani A. The Effect of Upper Limb Exercise on the Readmission Rate of Myocardial Infarc- } \\
\text { tion Patients. The Journal of Qazvin University of Medical Sciences. 2019; 23(3):226-237. https://doi.org/10.32598/JQUMS.23.3.226 }\end{array}$ \\
dol https://doi.org/10.32598/JQUMS.23.3.226
\end{tabular}

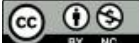

Received: 29 Sep 2018 Accepted: 19 Feb 2019 Available Online: 01 Aug 2019

Keywords: Upper extremity exercise, Admit, Myocardial infarction

\section{A B STRACT}

Background Myocardial infarction (MI) is among the most frequent causes of mortality and morbidity, with socioeconomic harms. Upper limb exercise, as part of cardiac rehabilitation program, is one of the secondary prevention methods of further $\mathrm{Ml}$ and disease improvement.

Objective The present study investigated the effect of upper limb sport on the rate of readmission in patients with myocardial infarction.

Methods In this randomized clinical trial study, 54 patients with $\mathrm{MI}$ were randomly divided into two groups of 27 patients (intervention and control). The intervention group performed upper extremity exercise for 6 weeks, twice a week and for 40 minutes per session. The demographic information of both groups was obtained before the exercise program, and at the end of the intervention. Furthermore, a checklist for referral to physicians and readmissions was completed. The collected data were analyzed using Independent Sample t-test and Chi-squared test.

Findings The number of readmission in the control group was higher than the test group; however, the group difference was not significant in terms of readmission $(P=0.250)$. Moreover, in terms of the reasons of readmission $(P=0.434)$, and the duration of readmission $(P=0.095)$, there was no significant difference between the two groups. The difference between the two groups in terms of the number of referral to physician $(P=0.231)$, and the reason for referral to physician, was not significant $(P=0.111)$. Conclusion The involvement of upper extremity exercises did not significantly change the rate of readmission and referral to physician of patients with myocardial infarction. Thus, further investigations are required in this regard.

\section{Extended Abstract}

\section{Introduction}

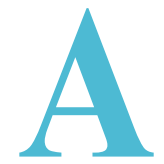

ccording to the Iran's Ministry of Health and Medical Education, cardiovascular diseases account for $45 \%$ of deaths [1]. Myocardial infarction (MI) is one of the most common and dangerous coronary heart diseases worldwide [2]. Car- diac rehabilitation is an ongoing and important care plan for patients with coronary artery disease [14]. Exercises and physical activities, as part of cardiac rehabilitation, provide individual and social health. Patients with MI should enroll in a supervised exercise program, along with clinical treatment [13]. The exercise program for patients with MI starts within 2-4 days after attack during hospitalization and 1421 days after discharge from hospital $[16,17]$.

\section{* Corresponding Author:}

Maryam Joodaki

Address: Department of Nursing, School of Nursing \& Midwifery, Qazvin University of Medical Sciences, Qazvin, Iran.

Tel: +98 (912) 4835424

E-Mail: m.joodaki@qums.ac.ir 
Upper extremity exercises are the aerobic program, which engages contraction and intermittent relaxation of large muscle groups. The purpose of these exercises is to stretch and strengthen the chest muscles and shoulder belts, which modify functional capacity, help in performing daily activities, reduce mortality and morbidity rate, improve quality of life, and reduce symptoms of activity intolerance and recurrence events [5]. Besides, upper extremity exercises can increase arterial blood pressure (compared to lower extremity exercises) and decrease stroke volume [26]. This study aims to evaluate the effect of upper extremity exercises in patients with MI.

\section{Materials and Methods}

In this randomized clinical trial study, conducted in 2017, a total of 54 patients with MI were recruited as the study samples using simple sampling technique. They were selected from those who referred to Velayat and Bou Ali Sina hospitals in Qazvin city, Iran. The inclusion criteria were as follows: aged 40-65 years; diagnosed with MI by a cardiologist; discharged 14-21 days ago; had left ventricular ejection fraction $<30 \%$ according to echocardiography; failed primary percutaneous coronary intervention; had a variety of uncomplicated MI except extensive MI confirmed by a cardiologist; and could exercise approved by a cardiologist.

They were randomly assigned into two groups of intervention $(n=27)$ and control $(n=27)$. The intervention group received supervised upper extremities exercises for 6 weeks, two sessions per week, each for $40 \mathrm{~min}$. At each session, before the exercises, the vital signs of the patients were monitored. Then warm-up stretching and light exercises were performed for 5 minutes. Afterward, the exercise movements of the upper extremities with a stick or 200-g weights for maintaining balance were performed for 30 minutes followed up by 5-min cooling up at the end. The patients performed each movement 5-15 times. There were fewer movements in the initial sessions and then the movements increased in the upcoming sessions. There were intervals of 2- to 3-minute short rest (depending on the situation of the patients) between movements. At the end of each session, the vital signs of patients were reassessed and recorded to ensure that they were in good condition. It should be noted that being absent for two sessions resulted in exclusion from the study. The control group performed routine care, and after the intervention, the study results were given to them.

Demographic information was collected from both groups before the intervention, and a checklist for a referral to physician and readmission was completed immediately after the 6-week intervention. The collected data were analyzed in SPSS V. 21 by using the Chi-squared test for testing the difference between groups in terms of the prevalence of readmission and referral to a physician, duration of readmission, and reasons for readmission and referral to a physician. The significance level was set at $\mathrm{P}<0.05$.

\section{Results}

The prevalence of hospital readmission at the end of the intervention for one-time hospitalization was $11.1 \%$ in the intervention group, and $18.5 \%$ in the control group, but the Chi-squared test results showed no significant difference between them $(\mathrm{P}=0.418)$. In the intervention group, 92.6\% had no readmission, while in the control group this figure was $77.8 \%$. The reasons for readmission were chest pain and shortness of breath $(3.7 \%)$. In the control group, the reasons were chest pain (7.4\%), shortness of breath $(7.4 \%)$, increased blood pressure $(3.7 \%)$ and side effects of the prescribed drugs (3.7\%). However, the difference between them was not significant $(\mathrm{P}=0.434)$.

The amount of readmission in the intervention group was $0 \%$ for $1-3$ days of hospitalization and $7.4 \%$ for hospitalization of more than 3 days. For the control group, it was $11.1 \%$ for both cases. The difference between them was not significant $(\mathrm{P}=0.095)$. The rate of referral to a physician in the intervention group for one visit was $70.4 \%$, and for two visits $7.4 \%$. For the control group, the rates were $51.9 \%$ and $22.2 \%$, respectively. There was also no significant difference between them $(\mathrm{P}=0.231)$. In the intervention group, $22.2 \%$ had no referral to a physician, while for the control group it was $25.9 \%$. The reasons for referral to a physician in the intervention group were follow up $(59.3 \%)$, chest pain $(11.1 \%)$, and shortness of breath $(7.4 \%)$. In the control group, the reasons were follow up $(29.6 \%)$, chest pain $(18.5 \%)$, shortness of breath (7.4\%), drug misuse (7.4\%), drug side effects $(7.4 \%)$, and increased blood pressure $(3.7 \%)$. The difference between them was not significant either $(\mathrm{P}=0.111)$.

\section{Conclusion}

The upper extremity exercises did not affect the rate of readmission and referral to a physician, duration of readmission, and reasons for readmission and referral to a physician. In the study of Zand et al. on the effect of rehabilitation programs for 6 months on the outcomes of MI, 39.47\% of the patients in the intervention group had hospital readmission due to cardiac disease or recurrence, while in the control group it was $48.64 \%$ [27]. Regarding the short duration of intervention in our study (6 weeks), their results are not consistent with ours. In the study of Santaularia et al. on the effect of the exercise training program on readmis- 
sions in patients with MI, the results showed that 3, 6, and 12 months after upper and lower extremities exercises, the readmission rate decreased by $12 \%$ [28]. In terms of the follow-up period and the type of exercise (a combination of upper and lower extremity exercises), their study was different from the present study.

\section{Ethical Considerations}

Compliance with ethical guidelines

This study was approved by the Ethics Committee of Qazvin University of Medical Sciences, Qazvin, Iran, and was registered in the Iranian Registry of Clinical Trials (Approval Code: IR.QUMS.REC.1396.110 and Registration Code: IRCT2017100936674N1).

Funding

This article is extracted from the MSc. thesis of Maryam Joodaki in Nursing in Qazvin University of Medical Sciences.

\section{Authors' contributions}

Conceptualization, editing, and writing: Leili Yekefallah; Writing draft: Maryam Joodaki; Methodology and conceptualization: Hamid Reza Javadi; and Data analysis: Ameneh Barikani.

Conflicts of interest

The authors declared no conflict of interest.

\section{Acknowledgements}

We want to thank all the colleagues and patients of Coronary care unit, Cardiology, and Cardiac Rehabilitation Clinic of Qazvin University of Medical Sciences, who helped us with this project. 


\title{
بررسى تأثير ورزش اندام فوقانى بر ميزان بسترى مجدد مبتلايان به سكته قلبى: يك مطالعه كارأزمايیىبالينى
}

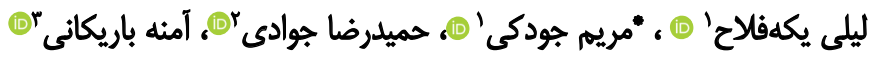

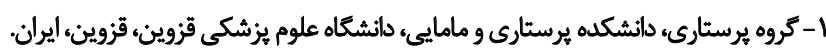

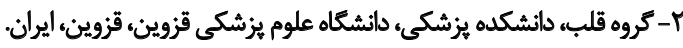

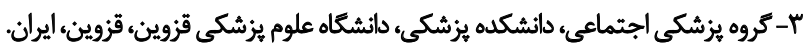

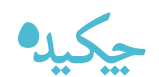

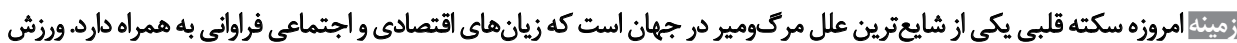

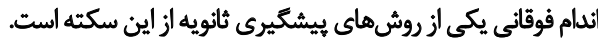

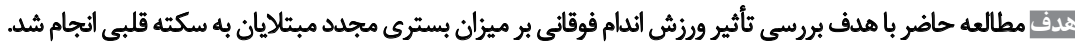

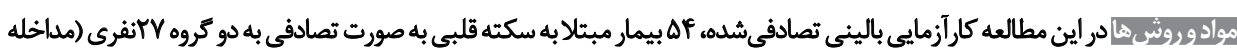

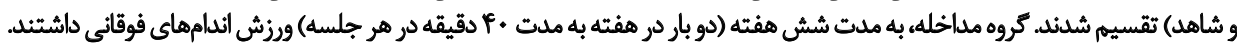

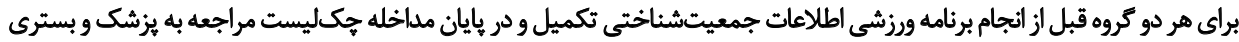

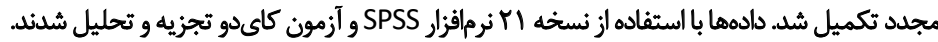

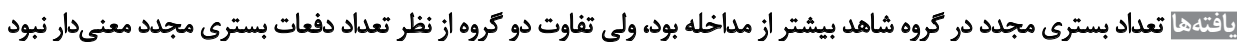

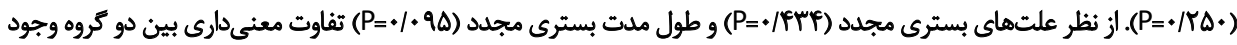

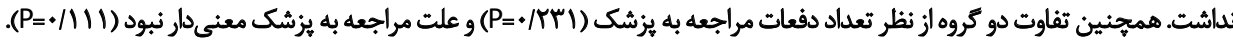

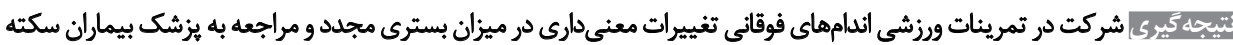

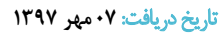

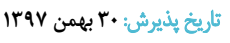

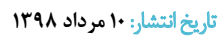

سكته قلبي سر آغاز مشكلات عديده قلبي است كه مدت ها يس

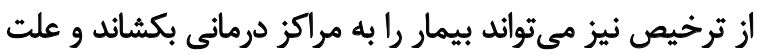

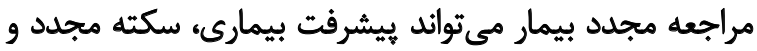

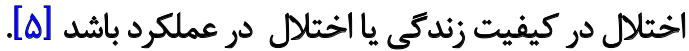

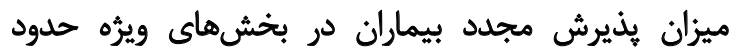

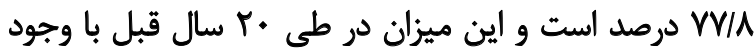

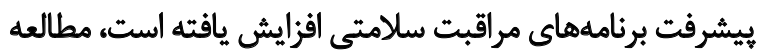

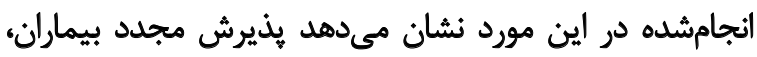

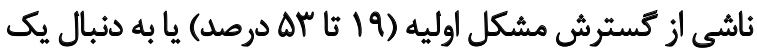

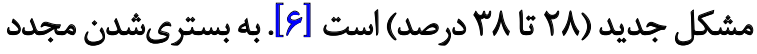

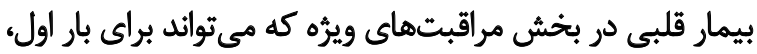

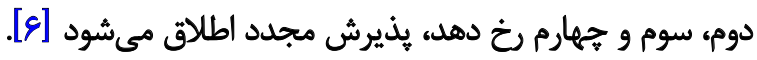

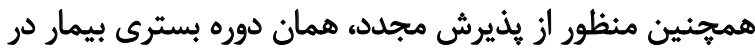

$\operatorname{dat} \%$

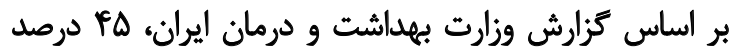

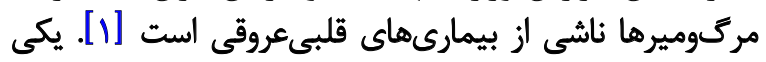

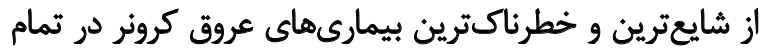

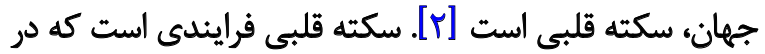

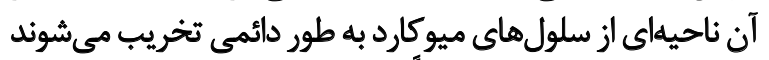

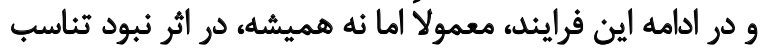

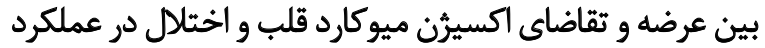

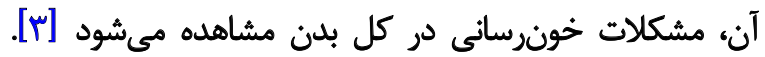

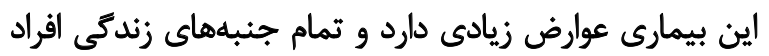

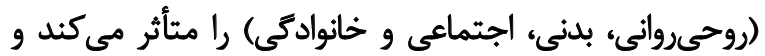

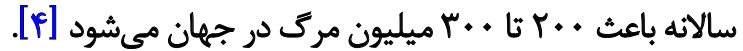

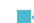

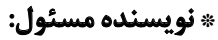

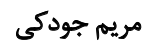

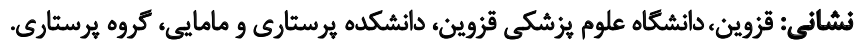
Pلفن: رايانامه: m.joodaki@qums.ac.ir 
تمرين ديناميك متداول، اغلب تحت عنوان "اتمرين تحملى"

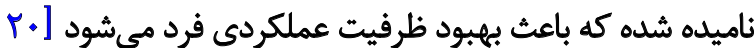

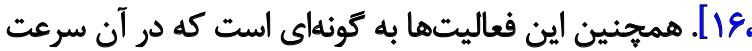

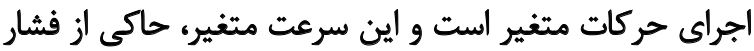

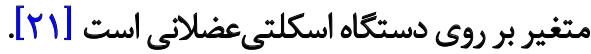
تمرينات هوازى قادرند توانايى عملكردى فرد را افزايش داده

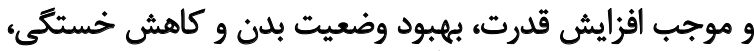

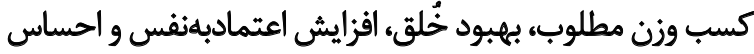

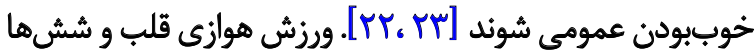

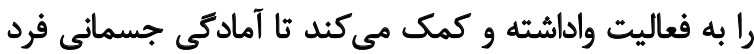

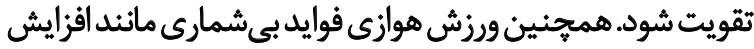

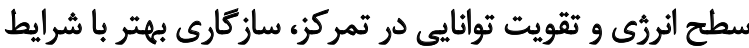

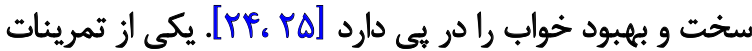

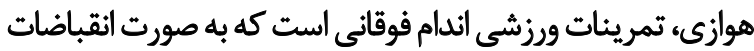

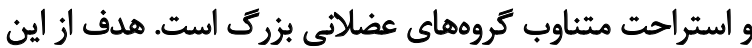

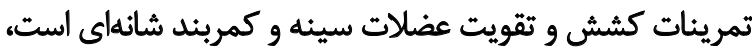

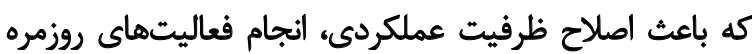

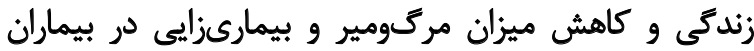

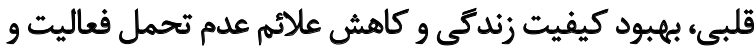

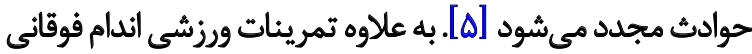

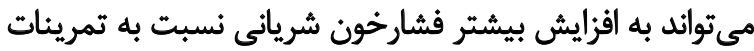

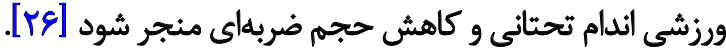
برنامه تمرينات ورزشى اندامهاى فوقانى در كشورمان در

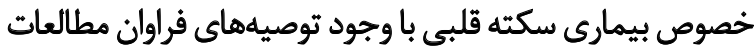

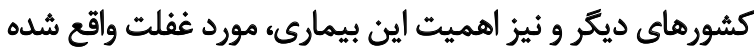

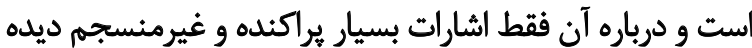

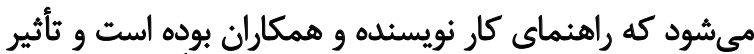

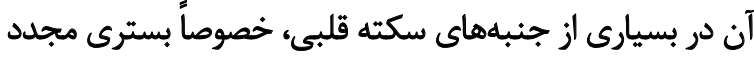

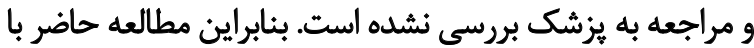

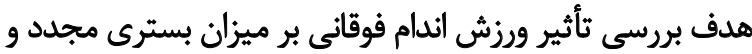

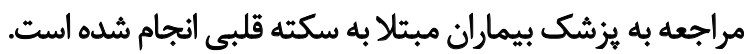

\section{Lus g glgo}

اين مطالعه كارآزمايى بالينى تصادفى به صورت نيمهتجربى

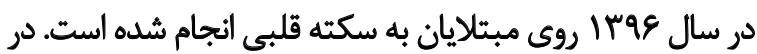

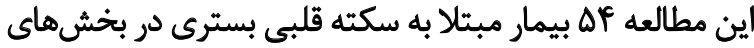

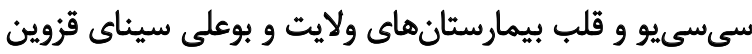

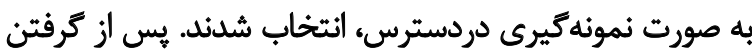

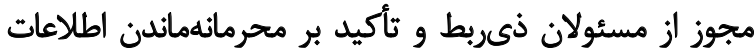

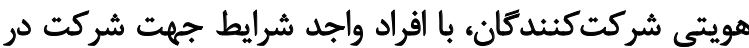

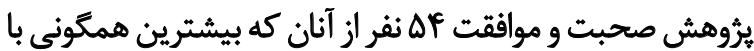

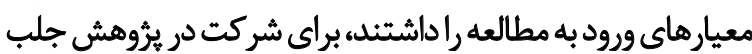

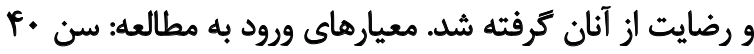

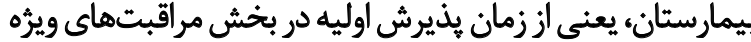

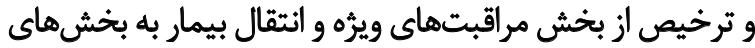

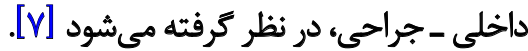

در مطالعات انجامشده تصور بر اين است كه ميزان بسترى مجدد إندا

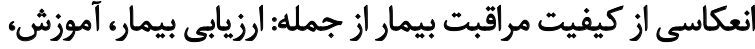

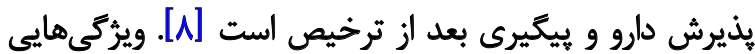

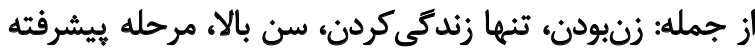

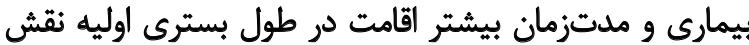

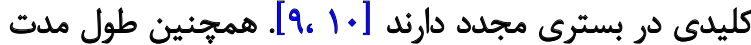

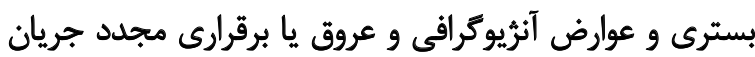

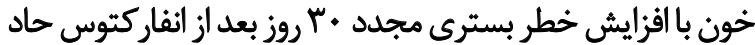

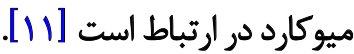

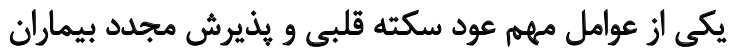

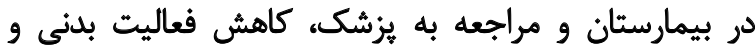

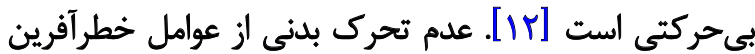

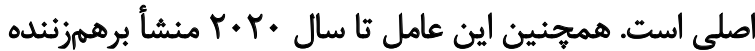

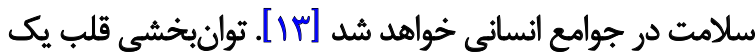

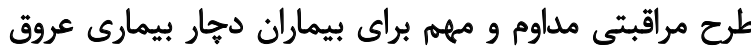

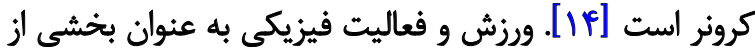

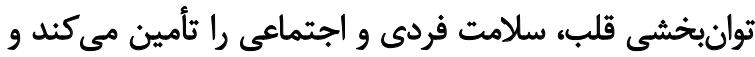

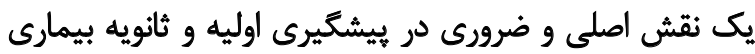

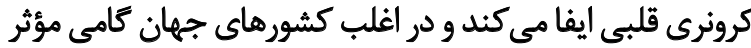

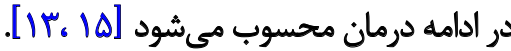

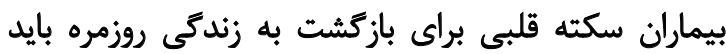

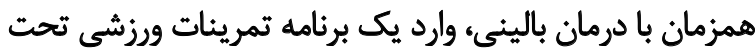

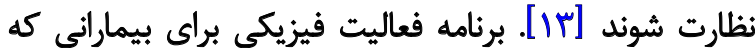

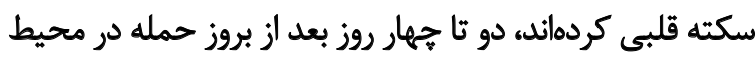

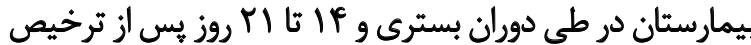
از بيمارستان انجام مي كيرد [19، IV]

يبيلى' و همكاران در نتايج يروهش خود شمارى از فوايد

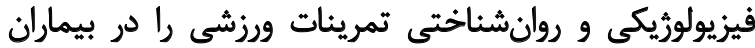

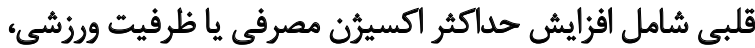
كاهش اكسيرن موردنياز عضله قلبى در هر سطح ازئ ازئ فعاليتهاي

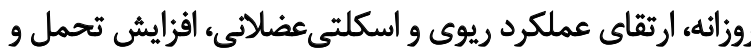

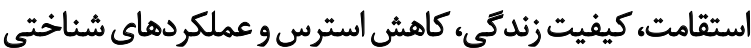

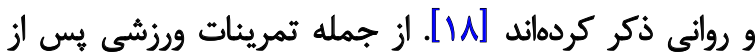

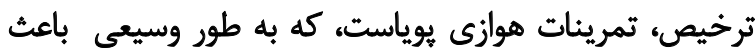

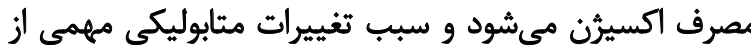

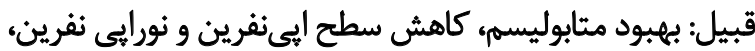

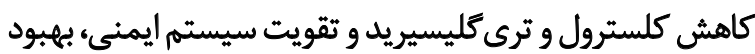
ترشح اندورفين و وضعيت خُلقى و وروانى مى تُشود [19] 
كوتاه Y تا بادقيقهاى (بر حسب نياز بيماران) بين حركات به آنها

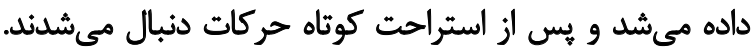

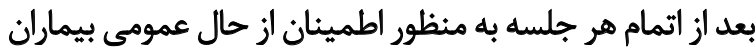

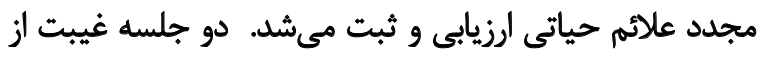
تمرينات باعث حذف آن فرد از مطالعه مى آشد.

كروه شاهد تحت مراقبثهاى معمول قرار كرفتند و وِ يس از

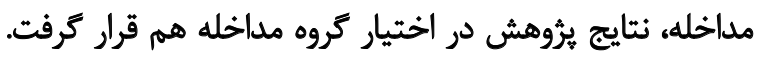

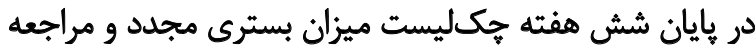
بf به يزشك جهت بيماران تكميل شد و بار ديكر اطلاعند

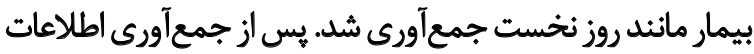

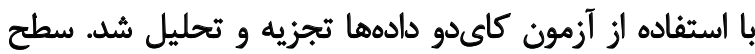

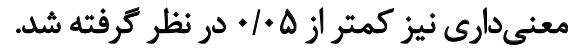

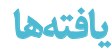

افراد از نظر سن، جنس، وضعيت تأهل، تحصيلات، مصرف

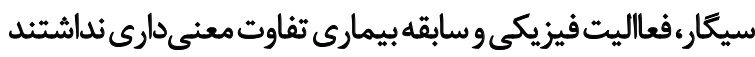

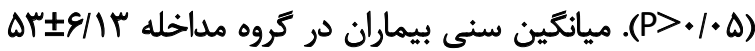

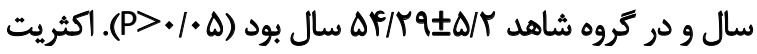

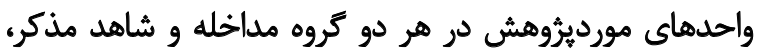

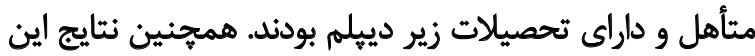

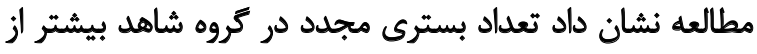

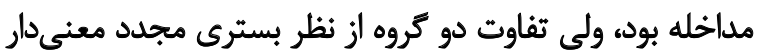

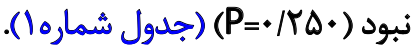

بررسى بيشتر نشان مىدهد علتهاى بسترى مجدد دري

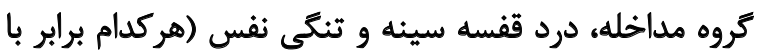

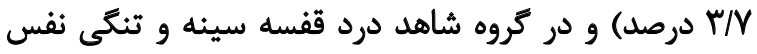

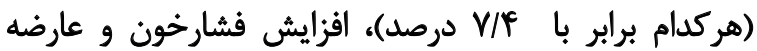

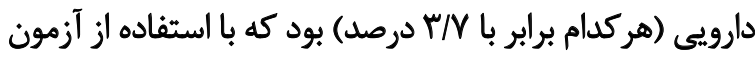

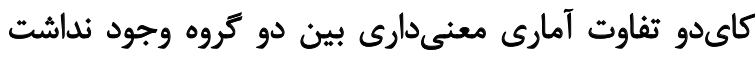

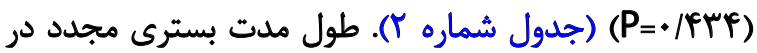

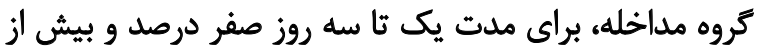

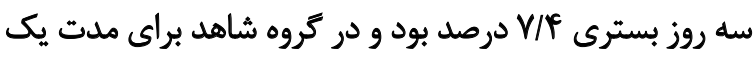

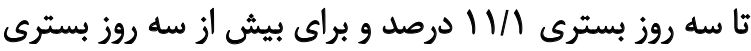

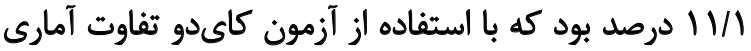

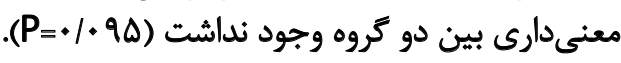

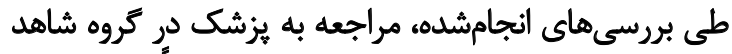

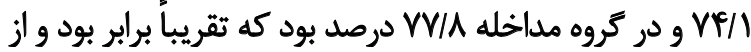
نظر آمارى تفاوت معنادار وجود نداشت مراش

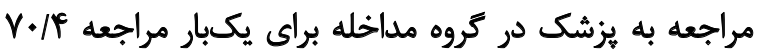

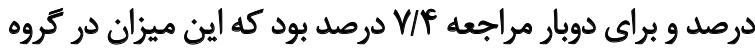

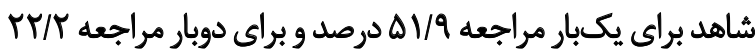

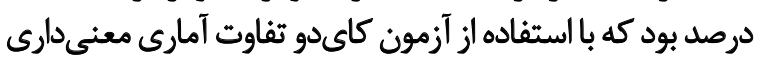

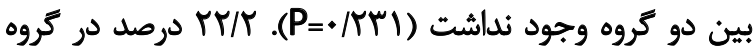

تا ه \& سال، تشخيص انفاركتوس قلبى طبق نظر متخصص قلب،

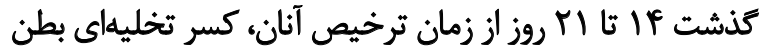

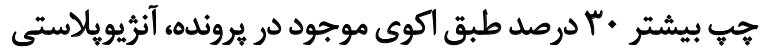

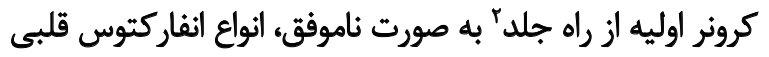

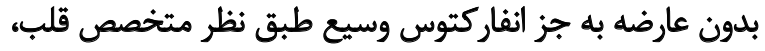
توانايى انجام ورزش طبق نظر متخصص قلب انب

بيماران در صورت داشتن شرايط زير از مطالعه خارج مى شيدند:

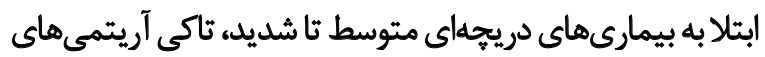

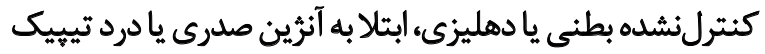

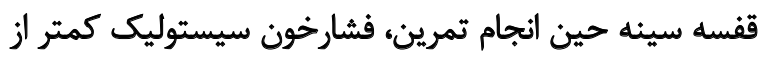

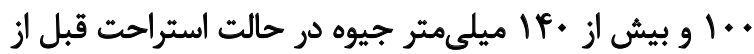

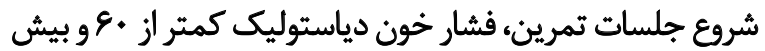

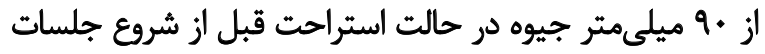

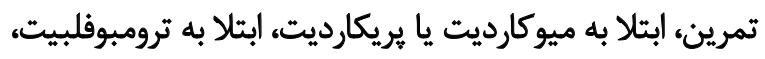

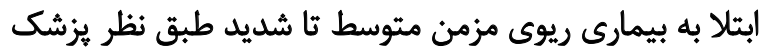

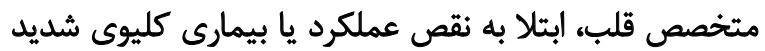

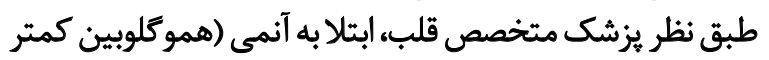

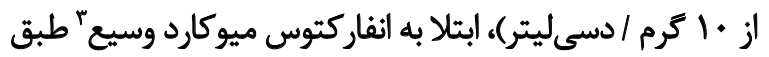
تشخيص متخصص قلب، آنزيويلاستى كرونرى اوليه موفق و ابتلائلا

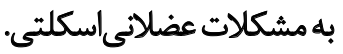

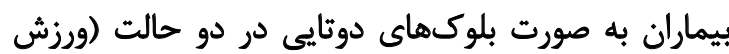

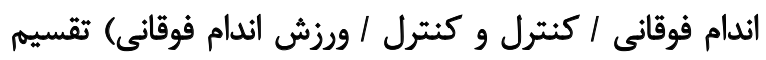

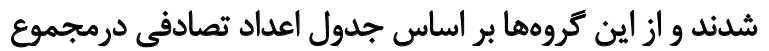

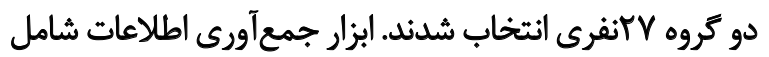

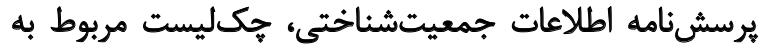

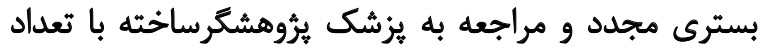

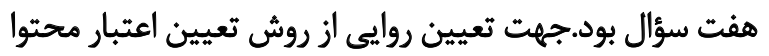

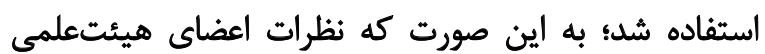

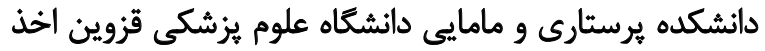

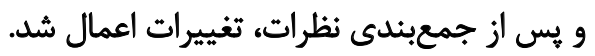

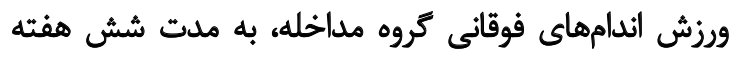

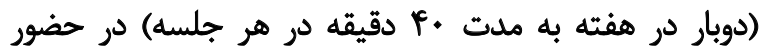

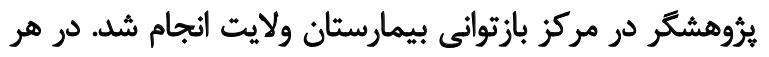

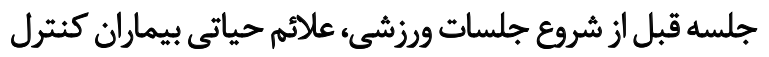

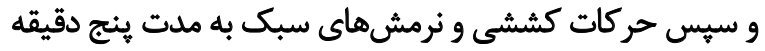

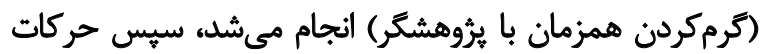

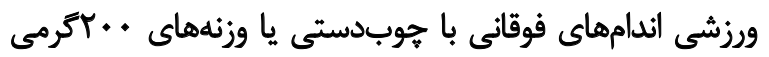

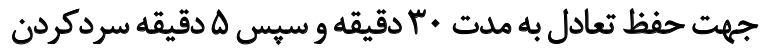

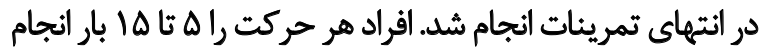

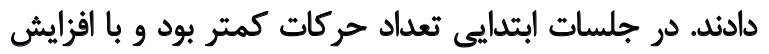

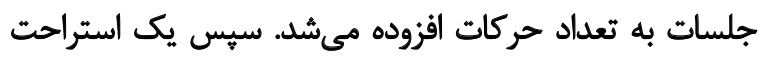

2. Primary percutaneous coronary intervention 3. Extensive myocardial infarction 
جدول (ا توزيع فراوانى بسترى مجدد شش هفته بعد از ترخيص

\begin{tabular}{|c|c|c|c|c|}
\hline جمع & خير & بلى & بسترى مجدد & كروه \\
\hline \multirow[t]{2}{*}{$r V$} & To & $r$ & تعلاد & \multirow{3}{*}{ ملاخليه } \\
\hline & & & & \\
\hline $1 .$. & $97 / 8$ & $V / P$ & ترود & \\
\hline \multirow[t]{2}{*}{ rV } & ri & 8 & تعلاد & \multirow{3}{*}{ شاهد } \\
\hline & & & & \\
\hline $1 .$. & $W / A$ & $r T / r$ & درهد & \\
\hline \multirow[t]{2}{*}{ af } & if & 1 & تعلاد & \multirow{3}{*}{ جمع } \\
\hline & & & & \\
\hline 1.0 & $A \Delta / T$ & $\| F / A$ & درصد & \\
\hline
\end{tabular}

مجلهملمى

كتستعلمعلوميزتنكى قزوين

جدول r. توزيع فراوانى بر حسب علت بسترى مجدد در دو كروه مداخله و شاهد بيماران مبثلا به سكته قلبى

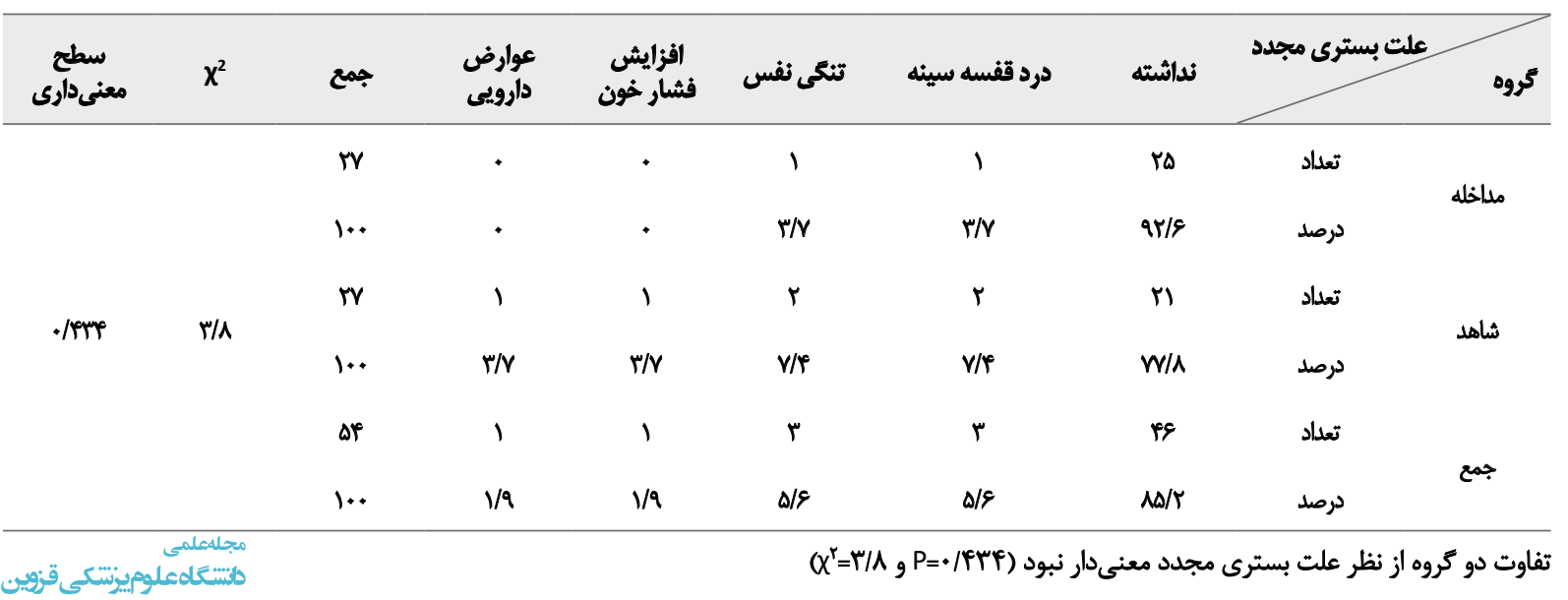

جدول با. توزيع فراوائى بر حسب علت مراجعه به يزشك در دو كروه مداخله و شاهد بيماران مبتلا به سكته قلبى

\begin{tabular}{|c|c|c|c|c|c|c|c|c|c|}
\hline جمع & عاروضيى & مصرف أشتباه & افزايش فشار & ديسينه & درد قفسه & جكاب & نداششه & علت مراجع & وه \\
\hline FV & - & - & • & $r$ & $r$ & 18 & 9 & تعلداد & \multirow[b]{2}{*}{ مداخله } \\
\hline 100 & - & - & - & $V / T$ & $m$ & $\Delta Q / r$ & $\pi / T$ & درصد & \\
\hline W & r & r & 1 & $r$ & $\Delta$ & $\wedge$ & $\checkmark$ & تعلاد & \multirow[b]{2}{*}{ شاهد } \\
\hline 1.0 & $V / s$ & $V / f$ & $r / Y$ & $V / f^{4}$ & WA & rq/e & $r a / q$ & درصد & \\
\hline of & r & r & 1 & $f$ & $\wedge$ & $\pi$ & ir & تعلاد & \multirow[b]{2}{*}{ جمع } \\
\hline 1.0 & $r N$ & $r / Y$ & 1/9 & $V / f$ & $1 f / A$ & $\mu f / f$ & $\pi / 1$ & درصد & \\
\hline \multicolumn{8}{|c|}{$x^{r}=91 . r, P=. / 111$} & \multicolumn{2}{|c|}{ نتيجه آزمون آمارى } \\
\hline
\end{tabular}

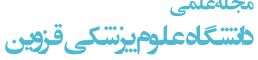


ورزشي اندام فوقاني و تحتاني، كاهش باهي الدرصدى بسترى هجدد

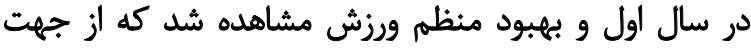

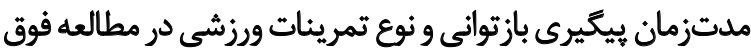

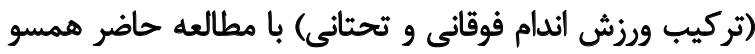

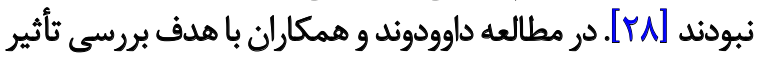
برنامههاي بازتوانى قلبى كوتاهمدت بر ميزان عود بيمارى، مراجعه داليه

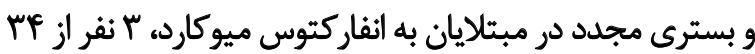

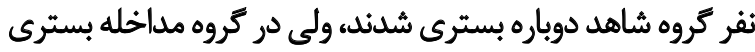

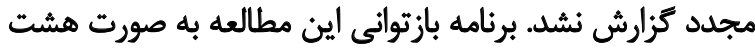

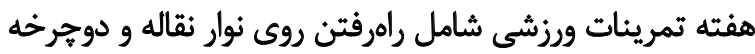

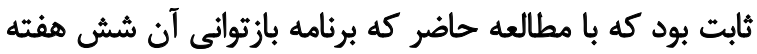

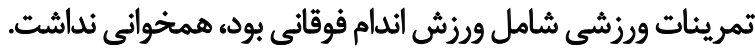

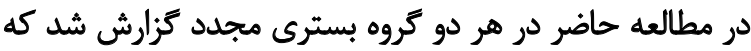

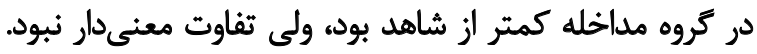

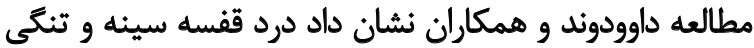

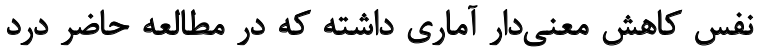

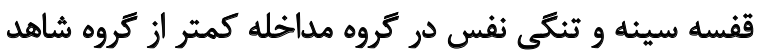
بود، ولى معنى دار نبود و نتايج مطالعه ما مطابق با نتايج مطالعه دئه

مذكور نبود [ه].

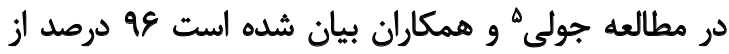

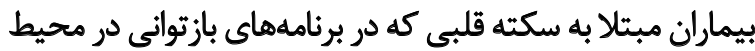

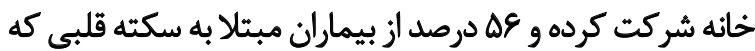

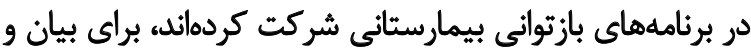

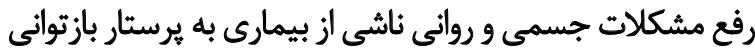

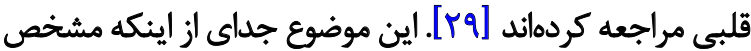
ميكند افرادى كه در برنامههاي منسجمثرتر شركت كردهانداند،

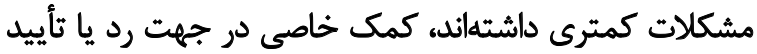

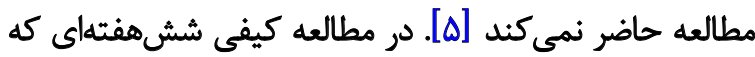

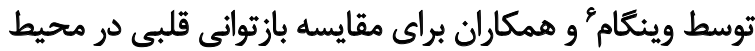

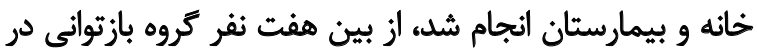

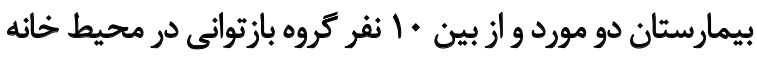

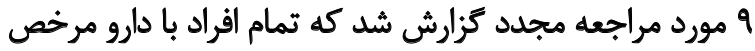

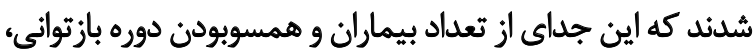

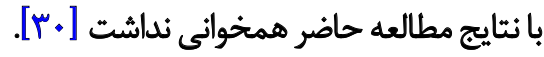

در يروهش حاضر، شركت در تمرينات ورزشى اندامهاى فوقانى

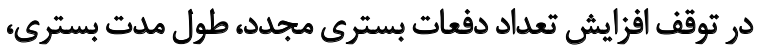
و علتهاى مراجعه به بيمارستان مؤثر بوده و حتى كاهش ميثى ميزان

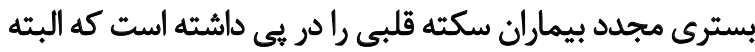

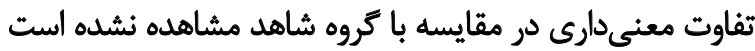

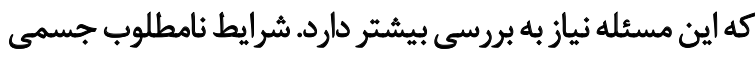

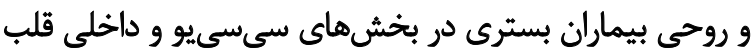

5. Jully
هداخله به يزشك هراجعه نداشتيند (يعنى بلون مراجعه)،

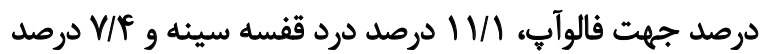

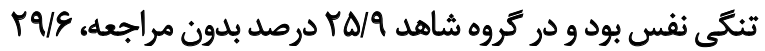

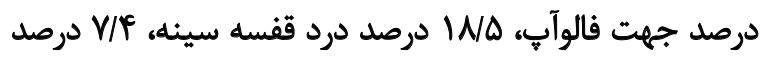

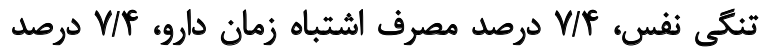

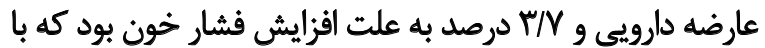

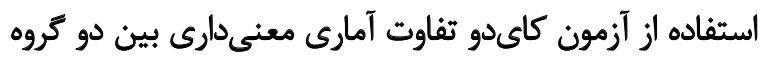

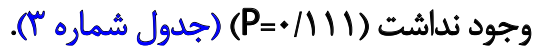

\section{s.s dow gen}

در برؤوهش حاضر ميزان بسترى مجدد در طي شش هفته

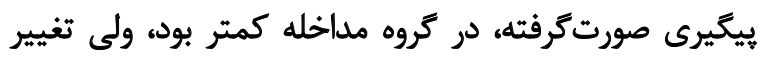

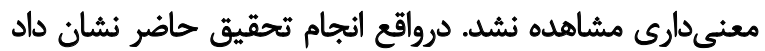

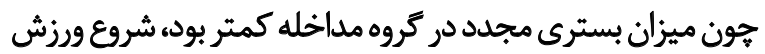

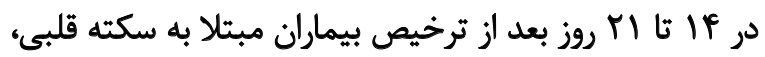

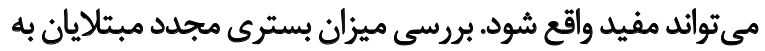

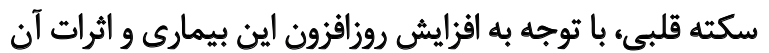

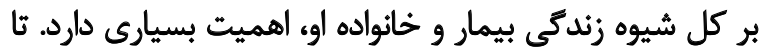

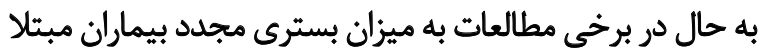

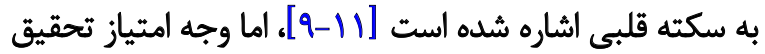

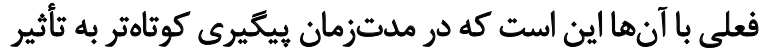

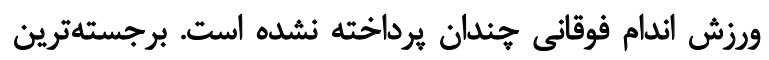

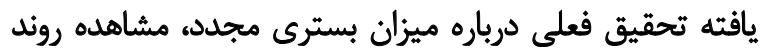

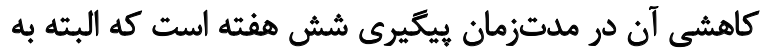

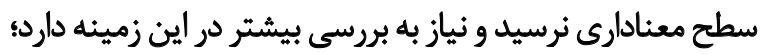

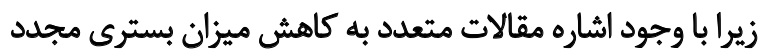

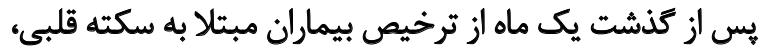

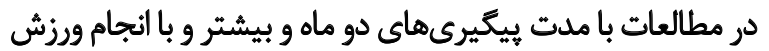

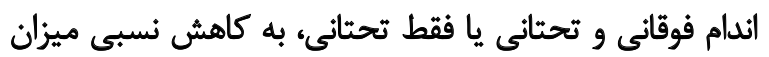

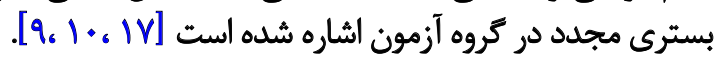
در مطالعه سليمان زند و همكاران كه با هدف بررسى تأثير

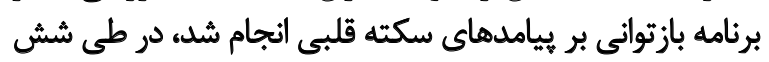

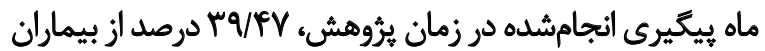

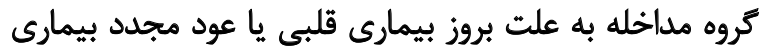

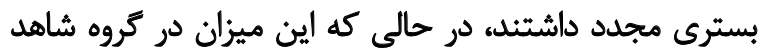

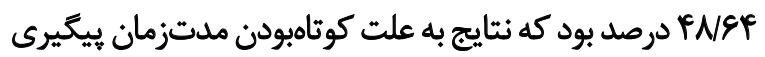
در طي شش هفته در مطالعه حاضر با يكديكر همخواني نداشت؛

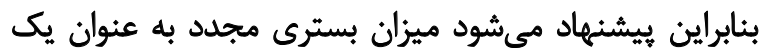

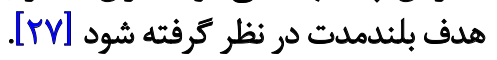
در مطالعه سانتاولارياّ و همكاران كه با هدف بررسى ثأثير

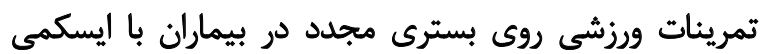

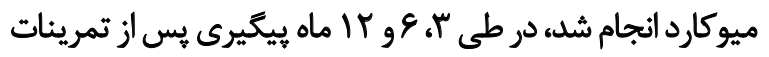


و مدتزمان كوتاه مطالعه از جمله محدوديتهاى يُزوهش

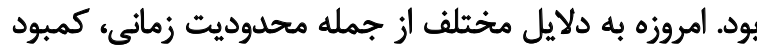

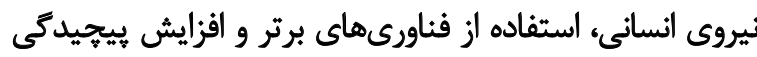

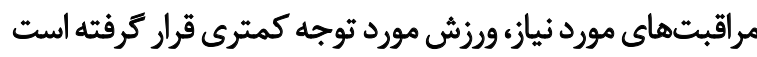

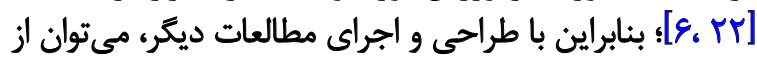

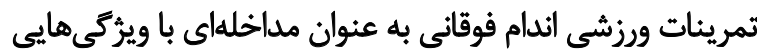

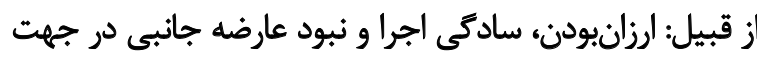

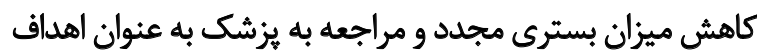
بلندمدت بهره برد.

مالاحظات اخالاقي

يميروى از اصول اخلاق يثوهش اين مطالعه با كد اخلاق IR.QUMS.REC.1396.110 در كر

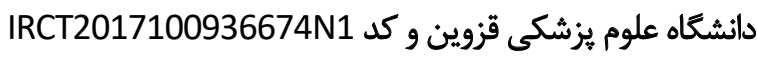
در بايكاه كارآزمايى بالينى به ثبث رسيده واست

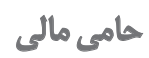

اين مقاله حاصل باياننامه مريم جودكي، دانشجوى

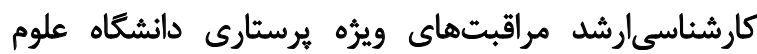

$$
\text { يزشكى قزوين است. }
$$$$
\text { مشار كت نويسندكًان }
$$

مفهومسازى، ويراستارى و نكارش: ليلى يكهفلاح؛ نكارش اوليه

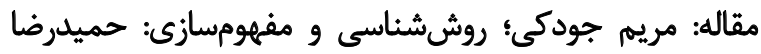
جوادى؛ تجزيه و تحليل دادهها: آمنه باريكاني.

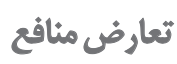

اين مطالعه هيجكونه تعارض منافعى نداشته است.

$$
\text { سباسكُز إرى }
$$

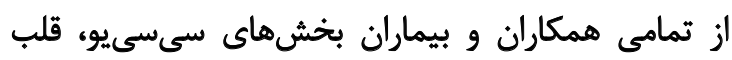

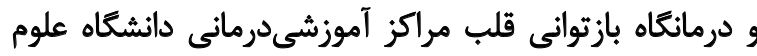
يزشكى قزوين كه در انجام اين طرح ما را يارى داني دادند، صميمانه

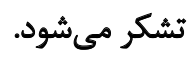




\section{References}

[1] Beyranvand MR, Lorvand A, Parsa SA, Motamedi MR, Kolahi AA. The quality of life after first acute myocardial infarction. Pajohandeh. 2011; 15(6):264-72. [In Persian]

[2] Mann DL, Zipes DP, Libby P, Bonow RO. Braunwald's heart disease e-book: A textbook of cardiovascular medicine. Edinburgh: Elsevier Health Sciences; 2014.

[3] Smeltzer Suzanne C. Brunner \& Suddarth's textbook of Medical-Surgical Nursing. [H Amini, Persian Trans.]. Tehran: Jameenegar; 2014

[4] Falvo DR. Effective patient evaluation: A guideto increased compliance. Boston: Jones and Batelfat; 2011.

[5] Davoudvand SM, Elahi N, Ahmadi F, Haghighi Zadeh MH. Effectiveness of the short-term cardiac rehabilitation programs on the rate of disease relapse, again refer and rehospitalization in patients with myocardial infarction. Rehab. 2008; 9(1):17-22. [In Persian]

[6] Tabannejad Z, Ebadi A. The role of nurses in reducing readmission to the intensive care unit patients. Q J Nurs Fac. 2015; 15(57):35-40. [In Persian]

[7] Kaben A, Correa F, Reinhart K, Settmacher U, Gummert J, Kalff $\mathrm{R}$, et al. Readmission to a surgical intensive care unit: Incidence, outcome and risk factors. Crit Care. 2008; 12(5):R123. [DOI:10.1186/cc7023] [PMID] [PMCID]

[8] Berenson A, Paulus RA, KalmanNS. Medicare's readmissionsreduction program-a positive alternative. N Engl J Med. 2012; 366(15):1364-6. [DOI:10.1056/NEJMp1201268] [PMID]

[9] Bradley EH, Curry L, Horwitz LI, Sipsma H, Thompson JW, Elma $\mathrm{M}$, et al. Contemporary evidence about hospital strategies for reducing 30-day readmissions: A national study. J Am Coll Cardiol. 2012; 60(7):607-14. [DOI:10.1016/j.jacc.2012.03.067] [PMID] [PMCID]

[10] Bettger JP, Alexander KP, Dolor RJ, Olson DM, Kendrick AS, Wing $L$, et al. Transitional care after hospitalization for acute stroke or myocardial zainfarction. Ann Intern Med. 2012; 157(6):407-16. [DOI:10.7326/0003-4819-157-6-20120918000004] [PMID]

[11] Dharmarajan K, Hsieh AF, Lin Z, Bueno H, Ross JS, Horwitz $\mathrm{LI}$, et al. Diagnoses and timing of 30-day readmissions after hospitalization for heart failure, acute myocardial infarction, or pneumonia. JAMA. 2013; 309(4):355-63. [DOI:10.1001/ jama.2012.216476] [PMID] [PMCID]

[12] Herliani YK, Rahayu U, Purba Cl, Harun H. Patients' needs on nutritional counseling and risk factor management among myocardial infarction patients in cardiac rehabilitation. J Nur Care. 2019; 2(2):104-10. [DOI:10.11591/.v5i1.4756]

[13] Naghibi S, Maleki J. The effect of exercise training on anaerobic threshold and exercise tolerance in patients with coronary artery disease-medical social. Social Research. 2011; 14(11):17-33. [In Persian]

[14] Hinkle J, Cheever KH. Brunner \& Suddarth's textbook of Medical-Surgical Nursing [Nikravan Mofrad M, Persian Trans.]. Tehran: Jamenegar; 2015.
[15] Perk J, De Backer G, Gohlke H, Graham I, Reiner Z, Verschuren $\mathrm{M}$, et al. European guidelines on cardiovascular disease preventation in clinical practice (version 2012). The fifth Joint task force of the European society on cardiovascular disease preventation in clinical practice (representatives of nine societies and by invited experts). Eur Heart J. 2012; 33(13):1635-701. [DOI:10.1093/eurheartj/ehs092] [PMID]

[16] Goushe B. New cardiac rehabilitation. Tehran: University of social Welfare \& Rehabilitation publishers; 2012. [In Persian]

[17] Nery RM, Zanini M, de Lima JB, Bühler RP, da Silveira AD, Stein R. Tai chi chuan improves functional capacity after myocardial infarction: A randomized clinical trial. Am Heart J. 2015; 169(6):854-60. [DOI:10.1016/j.ahj.2015.01.017] [PMID]

[18] Piepoli MF, Conraads V, Corra U, Dickstein K, Francis DP, Jaarsma $\mathrm{T}$, et al. Exercise training in heart failure: From theory to practice. A consensus document of the Heart Failure Association and the European Association for Cardiovascular Prevention and Rehabilitation. Eur J Heart Fail. 2011; 13(4):347-57. [DOI:10.1093/eurjhf/hfr017] [PMID]

[19] Martins RA, Veríssimo MT, e Silva MJ, Cumming SP, Teixeira AM. Effects of aerobic and strength-based training on metabolic health indicators in older adults. Lipids Health Dis. 2010; 9:76. [DOI:10.1186/1476-511X-9-76] [PMID] [PMCID]

[20] Ardestani A. Physical readiness. $2^{\text {nd }}$ Edition, Tehran: Gouyeshe No; 2014. [In Persian]

[21] Eil Beigi S, Ahmadi Broughani Y, Ramezani H, Bakhshi E. The calculating of isokinetic moment and comparison with isotonic moment in elbow flexion with ADAMS software. J Res Sport Rehab. 2013; 1(1):41-51. [In Persian]

[22] Yeh CH, Wai JP, Lin US, Chiang YC. A pilot study to examine the feasibility and effects of a home-based aerobic program on reducing fatigue in children with acute lymphoblastic leukemia. Cancer Nur. 2011; 34(1):3-12. [DOI:10.1097/ NCC.0b013e3181e4553c] [PMID]

[23] Lofrano-Prado MC, Hill JO, Silva HJ, Freitas CR, Lopes-de-Souza $S$, Lins TA, et al. Acute effects of aerobic exercise on mood and hunger feelings in male obese adolescents: A crossover study. Int J Behav Nutr Phys Act. 2012; 9:38. [DOI:10.1186/14795868-9-38] [PMID] [PMCID]

[24] Voet NB, Bleijenberg G, Padberg GW, van Engelen BG, Geurts AC. Effect of aerobic exercise training and cognitive behav ioural therapy on reduction of chronic fatigue in patients with facioscapulohumeral dystrophy: Protocol of the FACTS-2-FSHD trial. BMC Neurol. 2010; 10:56. [DOI:10.1186/1471-2377-1056] [PMID] [PMCID]

[25] Daei R, Jale Doust Sani B. Aerobic exercises: Cardiovascular enhancement. Tehran: Bamdad Book; 2011. [In Persian]

[26] Calbet JA, Gonzalez-Alonso J, Helge JW, Søndergaard H, Munch-Andersen T, Saltin B, et al. Central and periphera hemodynamics in exercising humans: Leg vs arm exercise. Scand J Med Sci Sports. 2015; 25(Suppl. 4):144-57. [DOI:10.1111/sms.12604] [PMID]

[27] Zand S, Kouhestani H, Baghcheghi N, Shah Mirzaei R. The effect of a rehabilitation programs on the effects of myocardial infarction. Nurs Res. 2011; 22(1):24-30. [In Persian] 
[28] Santaularia N, Caminal J, Arnau A, Perramon M, Montesinos J, Trapé J. Randomized clinical trial to evaluate the effect of a supervised exercise training program on readmissions in patients with myocardial ischemia: A study protocol. BMC Car Dis. 2013; 13(1):32. [DOI:10.1186/1471-2261-13-32] [PMID] [PMCID]

[29] Jully K, Lip G Y, Greefild S, Raftery J, Matt J. The birmingham rehabilitation uptake maximisation study (BRUM): Homebased copared with hospital- based cardiac rehabilitation in a multiethnic population: Cost- effectiveness and patient adherence. Health Tech Ass. 2007; 11(35):1-118. [DOI:10.3310/ hta11350]

[30] Wingham J, Dalal HM, Sweeney KG, Evans PH. Listening to patients: Choice in cardiac rehabilitation. Eur J Car Nurs. 2006; 5(2):289-94. [DOI:10.1016/j.ejcnurse.2006.02.002] [PMID] 
This Page Intentionally Left Blank 